\title{
Compact stars made of fermionic dark matter
}

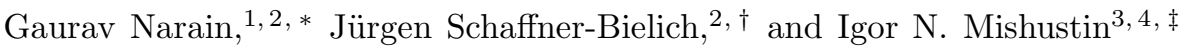 \\ 1 Indian Institute of Technology, Kanpur, Uttar Pradesh, India \\ ${ }^{2}$ Institut für Theoretische Physik, J. W. Goethe Universität, \\ Max von Laue-Straße 1, D-60438 Frankfurt am Main, Germany \\ ${ }^{3}$ Frankfurt Institute for Advanced Studies, J. W. Goethe Universität, \\ Max von Laue-Straße 1, D-60438 Frankfurt am Main, Germany \\ 4 The Kurchatov Institute, Russian Research Center, 123182 Moscow, Russia
}

(Dated: February 5, 2008)

\begin{abstract}
Compact stars consisting of fermions with arbitrary masses and interaction strengths are studied by solving the structure equation of general relativity, the Tolman-Oppenheimer-Volkoff equations. Scaling solutions are derived for a free and an interacting Fermi gas and tested by numerical calculations. We demonstrate that there is a unique mass-radius relation for compact stars made of free fermions which is independent of the fermion mass. For sufficiently strong interactions, the maximum stable mass of compact stars and its radius are controlled by the parameter of the interaction, both increasing linearly with the interaction strength. The mass-radius relation for compact stars made of strongly interacting fermions shows that the radius remains approximately constant for a wide range of compact star masses.
\end{abstract}

Keywords: compact stars, mass-radius relation, fermion stars, equation of state, dark matter

\section{INTRODUCTION}

Compact stars, white dwarfs and neutron stars, are one of the most enigmatic astrophysical objects (for an introduction to the physics of compact stars we refer to the excellent textbooks 1, 2, 3] and to recent pedagogical papers [4, 5, 6, 7, 8] ). The first successful description of compact stars was proposed by Fowler in 1926 [9], who first realized that Fermi-Dirac statistics is responsible for the high degeneracy pressure which holds up the star against gravitational collapse. Shortly afterwards Chandrasekhar applied effects of special relativity to the equation of state (EoS) of a degenerate Fermi gas and demonstrated the existence of a maximum mass for such stars beyond which they are unstable against collapse: the famous Chandrasekhar mass limit, $M_{c h} \approx 1.4 M_{\odot}[10]$.

In 1932 Chadwick discovered the neutron and Heisenberg presented his theory of isospin for nucleons suggesting that the neutron is a fermion with spin- $\frac{1}{2}$ like the electron. The ideas of Fowler and Chandrasekhar were then immediately extended to the case of degenerate neutrons and a new form of compact stars, neutron stars, was predicted by Landau 11]. The first numerical calculations for a neutron star within general relativity were performed by Oppenheimer and Volkoff in 1939 [12]. They computed a maximum stable mass of $0.75 M_{\odot}$ for a free Fermi gas of neutrons beyond which the star would be unstable and collapse into a black hole. The maximum mass limit for neutron stars is now well known as the Oppenheimer-Volkoff mass limit. Contrary to the case of the electron gas in white dwarfs, there are sizable contributions to the mass limit for neutron stars due to strong interactions between the neutrons. These interactions can be well modelled by an effective repulsive potential which can increase the maximum stable mass of a neutron star to about $3 M_{\odot}$. The presence of hyperons in the core of neutron stars leads to a softening of the equation of state and a reduction of the maximum mass [13, 14, 15, 16, 17].

As of today, new massive fermions are known within the standard model and many more have been predicted, in particular also as candidates for dark matter. In the year 1964, Gell-Mann and Zweig proposed the idea of quarks, putting forward the notion that neutrons and protons are composed of quarks. Ivanenko and Kurdgelaidze [18] studied a quark core in massive compact stars and Itoh 19] calculated the mass-radius relation of a quark star. If a compact star consists of quarks only, including besides the light quarks also the strange quark, they are dubbed strange stars 20, 21, 22]. Those exotic objects might be bound by strong interactions only, contrary to ordinary neutron stars and white dwarfs which are bound by gravity. The physics of compact stars with a quark core and strange stars is now a field of active research (for a recent review we refer to [23]) and has found its place in modern textbooks [1, 2]. Besides quarks, other fermions in the form of massive neutrinos are now well known to exist in nature. New types

\footnotetext{
*Electronic address: gauhari@iitk.ac.in

†Electronic address: schaffner@astro.uni-frankfurt.de

$\ddagger$ Electronic address: mishustin@fias.uni-frankfurt.de
} 
of fermions predicted in extensions of the standard model include the supersymmetric particles, the neutralino, the gravitino, and the axino, which are also candidates for dark matter (for a review see e.g. [24]).

Now the idea put forward by Fowler many years ago could be used to speculate on compact stars made out of exotic fermions, stabilised by the degeneracy pressure in the same way as in the case of neutron stars and white dwarfs. The present investigation is dealing with the following questions: What is the maximum stable mass of compact stars as a function of the fermion mass at zero temperature? What will happen if a repulsive interaction is included in the equation of this fermionic matter?

The paper is organised as follows: in section $\amalg$ we briefly recapitulate the structure equation for compact stars, the Tolman-Oppenheimer-Volkoff (TOV) equation. In section III we discuss general scaling solutions for compact stars with an arbitrary equation of state, in particular for a free Fermi gas and for an interacting Fermi gas. We show that Landau's estimate for the maximum mass and the corresponding radius is an exact scaling solution of the TOV equation for a free Fermi gas. Section IV is devoted to our numerical solution of the TOV equations. The equation of state for interacting fermions as well as extended scaling solutions of the TOV equations are discussed. We show that there is one unique mass-radius relation for compact stars made of free fermions if it is properly rescaled by the Landau mass and the Landau radius. We also find that for strongly interacting fermions the maximum mass and its radius are controlled by the interaction terms. The mass-radius relation changes such that there is a constant radius for a wide range of masses. Finally, in section $\nabla$ we summarise and discuss our findings.

\section{THE STRUCTURE EQUATION FOR COMPACT STARS}

Throughout this paper we will be dealing with models of compact stars where effects of general relativity are included for a consistent description as in the case of ordinary neutron stars. The typical mass of a neutron star is of the order of $\sim 1 M_{\odot}$ with typical radii of about $10 \mathrm{~km}$, i.e. $10^{-5} R_{\odot}$. Hence, the gravitational potential on the surface of neutron star will be $10^{5}$ times stronger compared to that of the Sun. Moreover, the corresponding Schwarzschild radius $r_{s}=2 G M / c^{2}$ is about $3 \mathrm{~km}$ in this case. Under such conditions, the curvature of space-time can not be ignored and general relativity is needed to describe the structure of such compact objects.

In order to find the structure of space-time created by the presence of a compact star one needs to solve the Einstein's field equations. For simplicity we assume that the metric is spherically symmetric and static, i.e. the Schwarzschild metric. In addition, the energy momentum tensor is assumed to be that of an ideal fluid,

$$
T_{\mu \nu}=p g_{\mu \nu}+(p+\rho) U_{\mu} U_{\nu}
$$

where $p$ and $\rho$ denote the pressure and energy density, and $U_{\mu}$ the four velocity of the fluid. Using the Einstein's field equation and the condition for hydrostatic equilibrium, $U_{\mu}=(1,0)$, one arrives at the following equations describing the structure of a compact star

$$
\begin{aligned}
\frac{d p}{d r} & =-\frac{G M \rho}{r^{2}}\left(1+\frac{p}{\rho}\right)\left(1+\frac{4 \pi r^{3} p}{M}\right)\left(1-\frac{2 G M}{r}\right)^{-1}, \\
\frac{d M}{d r} & =4 \pi r^{2} \rho
\end{aligned}
$$

which are just the Tolman-Oppenheimer-Volkoff (TOV) equations [12, 25, 26] (note, that throughout the paper, we are using natural units by setting $\hbar=c=1$ ). The detailed derivation of the equation (2) can be found in standard textbooks (see e.g. 1, 2, 27, 28]). This is the same equation of hydrostatic equilibrium as in the case of Newtonian gravity just modified by three correction factors (in the r.h.s.) due to effects of general relativity. The equation (3) simply defines the quantity $M(r)$, the amount of energy contained within the radius $r$.

The unknown functions in eq. (2) and eq. (3) are $\rho(r), p(r)$ and $M(r)$. For a given equation of state, relating $p$ and $\rho$, appropriate initial and boundary conditions are needed to solve the above set of equations. The radius of the star, $R$, is found by using the condition that the pressure vanishes at the surface of star. The mass $M(0)$ must be zero at $r=0$ and $M(R)$ gives the total mass of the star at $r=R$. The central pressure is calculated from the equation of state once the central energy density $\rho(0)=\rho_{0}$ is given as the initial condition.

\section{SCALING THE TOV EQUATION}

It is easy to see, that the TOV equation contains two essential dimensional quantities, $G$ which can be conveniently expressed in terms of the Planck mass as $G=M_{p}^{-2}$, and the fermion mass $m_{f}$ which characterises the equation of 
state. In this section, we show how the TOV equation can be transformed to scale-independent variables composed of $M_{p}$ and $m_{f}$. There are several reasons for such an approach. One reason is that the computational treatment of differential equations benefits from a dimensionless format. The other reason is that a scaled equation needs to be solved only once. As soon as the general solution is found one can just rescale it by appropriate (dimensionful) factors to get the result for specific (astro-)physical cases. The TOV equation can be scaled in the following ways.

\section{A. Landau's argument for deriving the maximum mass of compact stars}

Landau presented a very elegant argument for deriving the maximum mass of a compact star 11] (for details see also the treatises in [3, 29, 30]). He used only Newtonian gravity, special relativity and Fermi-Dirac statistics to estimate the maximum mass and the corresponding radius of a compact star.

For a star made of free fermions at zero temperature the Fermi momentum $k_{F}$ is related to number density $n$ by the following relation (below we suppress the dependence on the statistical degeneracy factor assuming $g=2$ )

$$
n=\frac{k_{F}^{3}}{3 \pi^{2}}=\frac{N}{4 \pi / 3 R^{3}}
$$

where $N$ is the total number of fermions in a star. For simplicity, here we consider a star of uniform number density and of radius $R$. Solving for $k_{F}$, one gets

$$
k_{F}=\left(\frac{9 \pi}{4}\right)^{1 / 3} \frac{N^{1 / 3}}{R} .
$$

The total mass of the star is given solely by the vacuum fermion mass, $m_{f}$, as

$$
M=m_{f} N
$$

Now, let us consider a fermion on the surface of the star. Its energy is given by

$$
E(R)=-\frac{G M m_{f}}{R}+\left(\frac{9 \pi}{4}\right)^{1 / 3} \frac{N^{1 / 3}}{R}
$$

where the first term gives the gravitational energy and the second term comes from the kinetic energy of an ultrarelativistic fermion on the top of the Fermi distribution. For small values of $R$ and negative energy $E$, the gravitational attraction overcomes the degeneracy pressure causing a collapse. For positive $E$ the degeneracy pressure exceeds the gravitational attraction and the star will expand until the particle density drops so much that $k_{F} \sim m_{f}$. For the non-relativistic gas the kinetic energy per particle is $3 k_{F}^{2} /\left(10 m_{f}\right)$, i.e. it changes with the radius as $R^{-2}$. This means that gravitation will finally prevail and the expansion will be terminated. As a result, a stable minimum will develop in $E(R)$.

A good estimate of the maximum possible number of fermions in a compact star can be obtained by considering the limiting case $E=0$, when the gravitational energy is exactly equal to the Fermi energy of the degenerate Fermi gas. Then we can express the maximum number of fermions as

$$
N_{\max }=\left(\frac{9 \pi}{4}\right)^{1 / 2} \frac{M_{p}^{3}}{m_{f}^{3}}
$$

The maximum mass of the star is obtained from the relation $M=m_{f} N$, hence

$$
M_{\max } \sim \frac{M_{p}^{3}}{m_{f}^{2}}
$$

An estimate for the corresponding radius of the maximum mass star can be obtained by assuming that the kinetic energy of the fermion on the surface is equal to its mass, i.e. at the border of becoming relativistic, $k_{F} \approx m_{f}$, which gives for the minimum radius of the star

$$
R_{m i n} \sim \frac{M_{p}}{m_{f}^{2}}
$$


For a neutron star, with the fermion mass taken to be that of the neutron $m_{n} \approx 1 \mathrm{GeV}$, the above relations give $M_{\max } \approx 1.63 M_{\odot}$ and $R_{\min } \approx 2.41 \mathrm{~km}$ which is a reasonable estimate. Note, that Landau's argument can be well applied also for white dwarfs. Here, one has to take care of the fact that the mass of the white dwarf is determined by the nucleon mass while the degeneracy pressure is provided by the electrons. Therefore, the maximum mass for white dwarfs, the Chandrasekhar mass limit, turns out to be similar to the one for neutron stars, while the radius increases by the ratio of the nucleon and the electron masses, i.e. by about a factor 2000 , to about $4000 \mathrm{~km}$ (all these values have to be corrected for the charge to mass ratio of nuclei, see e.g. [30]).

\section{B. The equation of state in dimensionless form}

The equation of state for a free gas of fermions at zero temperature $p(\rho)$ can be calculated via explicit expressions for the energy density and pressure:

$$
\begin{aligned}
\rho & =\frac{1}{\pi^{2}} \int_{0}^{k_{F}} k^{2} \sqrt{m_{f}^{2}+k^{2}} d k \\
& =\frac{m_{f}^{4}}{8 \pi^{2}}\left[\left(2 z^{3}+z\right)\left(1+z^{2}\right)^{\frac{1}{2}}-\sinh ^{-1}(z)\right] \equiv m_{f}^{4} \rho^{\prime} \\
p & =\frac{1}{3 \pi^{2}} \int_{0}^{k_{F}} \frac{k^{4}}{\sqrt{m_{f}^{2}+k^{2}}} d k \\
& =\frac{m_{f}^{4}}{24 \pi^{2}}\left[\left(2 z^{3}-3 z\right)\left(1+z^{2}\right)^{\frac{1}{2}}+3 \sinh ^{-1}(z)\right] \equiv m_{f}^{4} p^{\prime}
\end{aligned}
$$

using natural units of $m_{f}^{4}$ and defining the relativity parameter $z=k_{F} / m_{f}$.

We introduce now the following dimensionless quantities for the mass and the radius of the star,

$$
M^{\prime}=\frac{M}{M_{L}} \quad \text { with } \quad M_{L}=\frac{M_{p}^{3}}{m_{f}^{2}} \quad \text { and } \quad r^{\prime}=\frac{r}{R_{L}} \quad \text { with } \quad R_{L}=\frac{M_{p}}{m_{f}^{2}}
$$

where $M_{L}$ and $R_{L}$ denote the maximum mass and corresponding radius as given by Landau's arguments (note that $R_{L}$ is equal to half the Schwarzschild radius). Using the above definitions and substituting them into eq. (2) and eq. (3) with $G=M_{p}^{-2}$, one obtains the following dimensionless form of the TOV equations:

$$
\begin{aligned}
\frac{d p^{\prime}}{d r^{\prime}} & =-\frac{M^{\prime} \rho^{\prime}}{r^{\prime 2}}\left(1+\frac{p^{\prime}}{\rho^{\prime}}\right)\left(1+\frac{4 \pi r^{\prime 3} p^{\prime}}{M^{\prime}}\right)\left(1-\frac{2 M^{\prime}}{r^{\prime}}\right)^{-1} \\
\frac{d M^{\prime}}{d r^{\prime}} & =4 \pi r^{\prime 2} \rho^{\prime}
\end{aligned}
$$

In general, the equation of state can not be expressed in a simple polytropic form $p^{\prime} \propto \rho^{\prime \gamma}$ with a constant $\gamma$. However, this can be done for two limits of the relativity parameter $z$. In the non-relativistic limit, $z \ll 1$, we get a polytropic law with $\gamma=5 / 3$, i.e.

$$
p^{\prime} \propto \rho^{\prime 5 / 3} \quad(z \ll 1)
$$

In the ultra-relativistic limit, $z \gg 1$, the equation of state approaches a polytrope of $\gamma=1$, i.e.

$$
p^{\prime}=\frac{\rho^{\prime}}{3} \quad(z \gg 1) .
$$

As the equation of state is a function of the relativity parameter $z$, one can compute it parametrically in a tabular form for a desirable interval of $z$. Fig. 11 depicts the resulting dimensionless pressure versus the dimensionless energy density in a double logarithmic plot. The curve exhibits two different slopes for small and large values of $\rho^{\prime}$. The larger slope at small values of $\rho^{\prime}$ shows the non-relativistic regime, the smaller slope at larger values of $\rho^{\prime}$ the relativistic regime as expected.

For the case of white dwarfs, the following changes have to be made to the equation of state, eq. (11) and eq. (12):

1. As the pressure is given by the degeneracy pressure of electrons, the expression for the pressure remains the same where $m$ is the mass of the electron. 


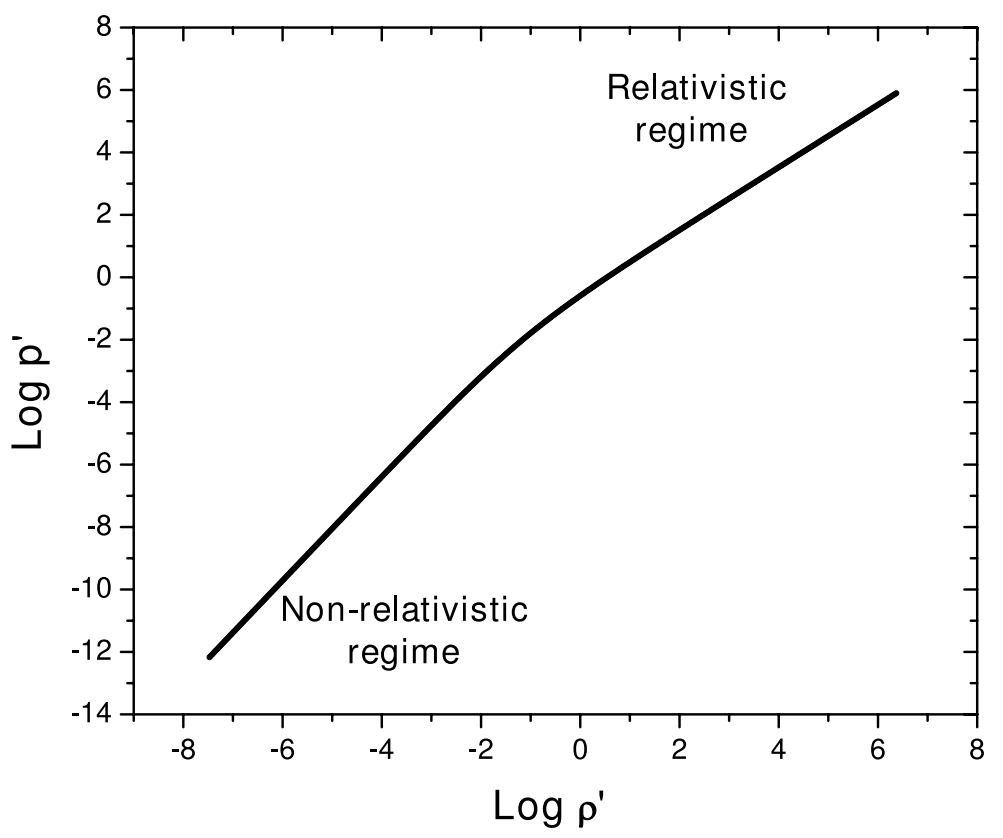

FIG. 1: The equation of state, the dimensionless pressure $p^{\prime}$ versus the dimensionless energy density $\rho^{\prime}$ for a free Fermi gas at zero temperature. The two limits, the non-relativistic one for $z \ll 1$ and the ultra-relativistic one for $z \gg 1$, can be clearly seen.

2. The energy density is a sum of two terms, one coming from the mass density of protons and neutrons while the other from the kinetic energy of electrons.

For a white dwarf consisting of nuclei with the atomic mass $A$ and atomic number $Z$ the mass density of protons and neutrons reads (small effects from the nuclear binding energy are disregarded here)

$$
\rho_{1}=n \cdot m_{N} \cdot \frac{A}{Z}
$$

where due to charge neutrality the number density of protons $n$ is equal to the number density of electrons which is given by eq. (4). The total energy density is now written as

$$
\rho=\rho_{1}+\frac{m_{e}^{4}}{\pi^{2}} \int_{0}^{z} x^{2} \sqrt{1+x^{2}} d x
$$

The second term here gives the relativistic energy density of electrons (note that here $z=k_{F} / m_{e}$ ). The resulting equation of state can be scaled in the same way as done before. Defining the dimensionless pressure and dimensionless energy density as $p^{\prime}=p / m_{e}^{4}$ and $\rho^{\prime}=\rho / m_{e}^{4}$, we can rewrite the above equation in the dimensionless form

$$
\rho^{\prime}=\frac{z^{3}}{3 \pi^{2}} \frac{m_{N}}{m_{e}} \frac{A}{Z}+\frac{1}{\pi^{2}} \int_{0}^{z} x^{2} \sqrt{1+x^{2}} d x .
$$

The pressure $p^{\prime}$ is given by eq. (12) where $m_{f}=m_{e}$. In the non-relativistic case, $z \ll 1$, the equation of state simplifies to eq. (16) . On the other hand, in the relativistic limit $1 \ll z \ll 6 \cdot 10^{3}$, when the first term on the r.h.s. dominates, the equation of state for white dwarfs becomes

$$
p^{\prime} \propto \rho^{\prime 4 / 3} .
$$

The proportionality constant in both limits, non-relativistic and relativistic ones, depends on $A$ and $Z$. Note, that the special case for white dwarfs emerges due to the presence of oppositely charged fermions which are neutralising each other. The heavier one determines the energy density, while the lighter one the pressure. For a pure charge-neutral Fermi gas, as we will consider later, the ultra-relativistic limit will be realized, as for the case of neutron stars. 


\section{Scaling solution for self-bound compact stars}

Another scaling solution is well known [20, 21] for the special form of the equation of state (see also [1] and references therein):

$$
p=s\left(\rho-\rho_{0}\right)
$$

where $s$ and $\rho_{0}$ are constants. Note, that this equation of state has a vanishing pressure at a finite energy density $\rho=\rho_{0}$. This property allows for the existence of self-bound balls of any size, not necessarily of astronomical scale. These balls are stabilised by other interactions not by gravity as in the case of neutron stars.

The scaling relations $p^{\prime}=p / \rho_{0}, \rho^{\prime}=\rho / \rho_{0}, r^{\prime}=\sqrt{\rho_{0}}\left(r / M_{P}\right)$ and $M^{\prime}=\sqrt{\rho_{0}}\left(M / M_{P}^{3}\right)$ change the TOV equation to a dimensionless form. One can then solve this equation numerically. If the mass and the radius are known for some particular value of $\rho_{0}$, then for some other value $\rho_{0}^{\prime}$ the radius and mass will be $R\left(\rho_{0}^{\prime}\right)=\sqrt{\rho_{0}^{\prime} / \rho_{0}} R\left(\rho_{0}\right)$ and $M\left(\rho_{0}^{\prime}\right)=\sqrt{\rho_{0}^{\prime} / \rho_{0}} M\left(\rho_{0}\right)$, respectively. Hence, both the mass and the radius scale with $1 / \sqrt{\rho_{0}}$.

The above equation of state is actually the one of the MIT bag model often used for describing cold and massless (strange) quark matter. The corresponding self-bound compact stars are dubbed strange stars 20, 21, 22]. Most studies of quark stars and strange stars utilise the equation of state derived from the MIT bag model. Ignoring effects from a finite quark mass, this model gives the equation of state

$$
p=\frac{1}{3}(\rho-4 B)
$$

where $B$ is the bag constant. Interestingly, the equation of state for an interacting cold gas of massless quarks within perturbative quantum chromodynamics can be approximated by the same form of the equation of state [31]. Standard values for the MIT bag constant are around $B^{1 / 4}=145 \mathrm{MeV}$ as follows from fits to hadron masses, which results in maximum masses of about $2.0 M_{\odot}$ at a radius of about $11 \mathrm{~km}$ [20, 21, 32], which are actually very close to the ones of realistic neutron star models. It is worth noting, that these values of $B$ are obtained in fits including the chromomagnetic interaction. In simplified versions of this model, disregarding the interaction effects, higher values, $B^{1 / 4} \simeq 200 \mathrm{MeV}$, are needed to preserve the stability of normal nuclear matter 33 .

\section{Scaling in a general case}

Consider the TOV equation for the pressure, eq. (2). We make the observation that the three correction factors from general relativity are already in a dimensionless form. Therefore, from the first factor $(1+p / \rho)$ one can scale pressure and energy density by a common factor $\epsilon_{\circ}$ as $p^{\prime}=p / \epsilon_{\circ}$ and $\rho^{\prime}=\rho / \epsilon_{\circ}$, respectively. Similarly, one defines a dimensionless mass and radial coordinate via $M^{\prime}=M / a$ and $r^{\prime}=r / b$. Plugging these definitions into the second factor $\left(1+4 \pi r^{3} p / M\right)$ one arrives at a dimensionless number $b^{3} \epsilon_{\circ} / a$ which we equate to one. Similar reasoning for the third factor $(1-2 G M / r)^{-1}$ results in $a /\left(M_{p}^{2} b\right)$ as the dimensionless number which again is equated to one. So we get finally the following scaling conditions

$$
\frac{b^{3} \epsilon_{\circ}}{a}=1 \quad \text { and } \quad \frac{a}{M_{p}^{2} b}=1
$$

Solving for $a$ and $b$ from the above equations one gets the following expressions

$$
a=\frac{M_{p}^{3}}{\sqrt{\epsilon_{\circ}}} \quad \text { and } \quad b=\frac{M_{p}}{\sqrt{\epsilon_{\circ}}} .
$$

For a free gas of fermions at zero temperature, we know from the direct calculation that $\epsilon_{\circ}=m_{f}^{4}$ (see eq. 11). Substituting this value of $\epsilon_{\circ}$ into the above equations one finds $a=M_{p}^{3} / m_{f}^{2}$ and $b=M_{p} / m_{f}^{2}$ which are exactly the same scaling factors as used originally by Landau. In the case of the MIT bag equation of state, the scaling factors are given by eqs. 25] with $\epsilon_{\circ}=\rho_{0}=4 B$.

We note in passing that according to Landau's argument it is sufficient to incorporate special relativity and Newtonian gravity to obtain the maximum mass $M_{\max }$ and the corresponding minimum radius $R_{\min }$ of a cold compact star made of fermions. Interestingly, the same dimensional forms for the maximum mass and minimum radius are found when using dimensional reasoning applied to the full TOV equation of general relativity. 


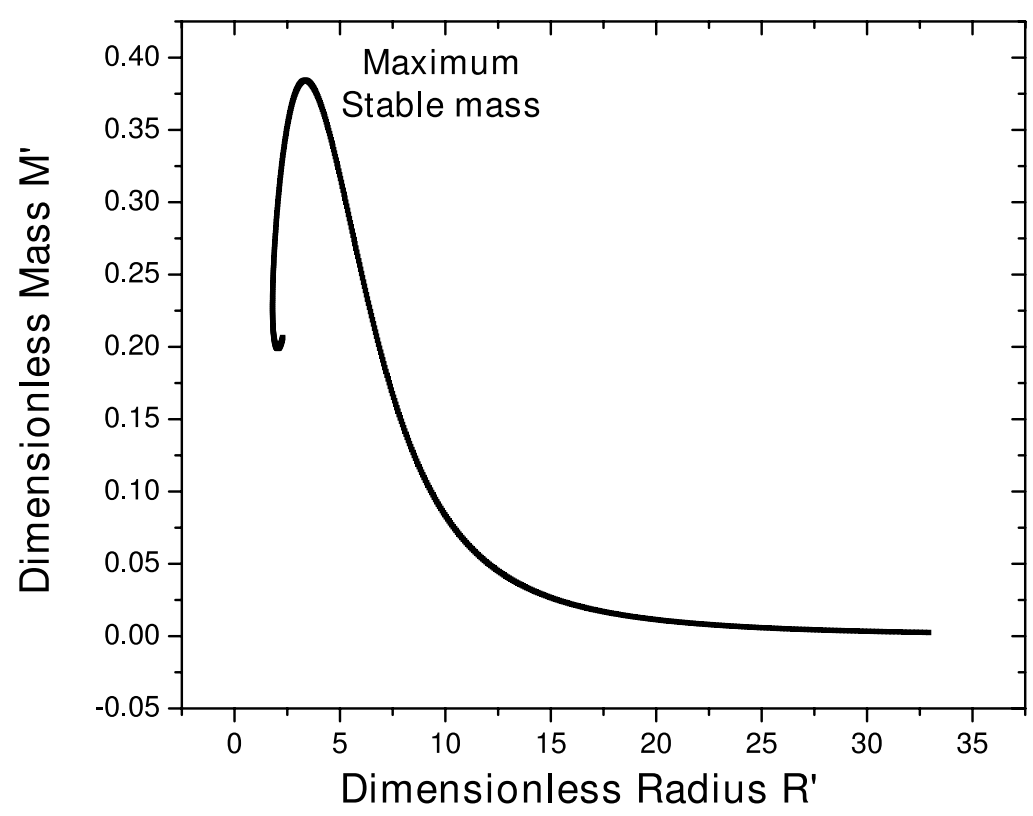

FIG. 2: Mass-radius relation in Landau units obtained by solving numerically the dimensionless TOV equation for a range of dimensionless central densities from $\rho^{\prime}=10^{-8}$ to $\rho^{\prime}=10$. Note, that the curve does not depend on the mass of the fermions forming the compact star.

\section{NUMERICAL SOLUTION OF THE TOV EQUATION}

\section{A. Stars made of free fermions}

For a free gas of fermions at zero temperature, the equations to be solved are the dimensionless TOV eq. (14) and eq. (15) along with the dimensionless equation of state given in parametric form by equations (11) and (12). One starts with the dimensionless central pressure or energy density, then solves the TOV equations from the center of the star to the surface where the pressure becomes zero. The corresponding radial distance defines the radius of the compact star.

Since the dimensionless equation of state is given as a function of parameter $z$, both quantities $p^{\prime}$ and $\rho^{\prime}$ are calculated for various values of $z$ and expressed in a tabular form. To calculate $p^{\prime}$ for a given $\rho^{\prime}$ and vice-versa, we use a simple linear interpolation. The dimensionless TOV equation is solved using a fourth-order Runge-Kutta algorithm. To plot the $M^{\prime}$ versus $R^{\prime}$ curve, $M^{\prime}$ and $R^{\prime}$ are calculated for various dimensionless central densities. The step size of the dimensionless radius lies between 0.01 (for very low dimensionless central density) and 0.001 (for very high dimensionless central density) to have at least 2000 points for one star configuration. The step size in the dimensionless central density is adjusted to have 500 points between $10^{-8}$ and 10 . The final dimensionless mass-radius relation is plotted in Fig. 2]

From the curve in Fig. 2 one notes that for a small dimensionless mass the dimensionless radius is large. This behaviour occurs for very small dimensionless central densities. The gravitational attraction is small in this case making the dimensionless radius large. As the dimensionless central density increases, the mass of the star increases, too. This leads to a stronger inward gravitational pull and smaller dimensionless radii. Thus, as we increase the dimensionless central density the mass increases while the radius decreases. A maximum mass is reached for the dimensionless mass $M_{\max }^{\prime}=0.384$ at the dimensionless radius $R_{\min }^{\prime}=3.367$. The presence of the maximum in the curve is generic and expected for an arbitrary fermion mass. The reason is that the energy density which generates the gravitational pull inwards has to be balanced by the outward fermionic pressure. However, the rate of change of the pressure with energy density is related to the speed of sound which is bounded by the speed of light. This speed limit puts a bound on the pressure increment with respect to changes in energy density. Thus an increase of the central energy density results in an increased gravitational attraction which cannot be compensated by the corresponding additional pressure, that leads eventually to a maximum mass limit [10, 11]. It is easy to see [3], that 


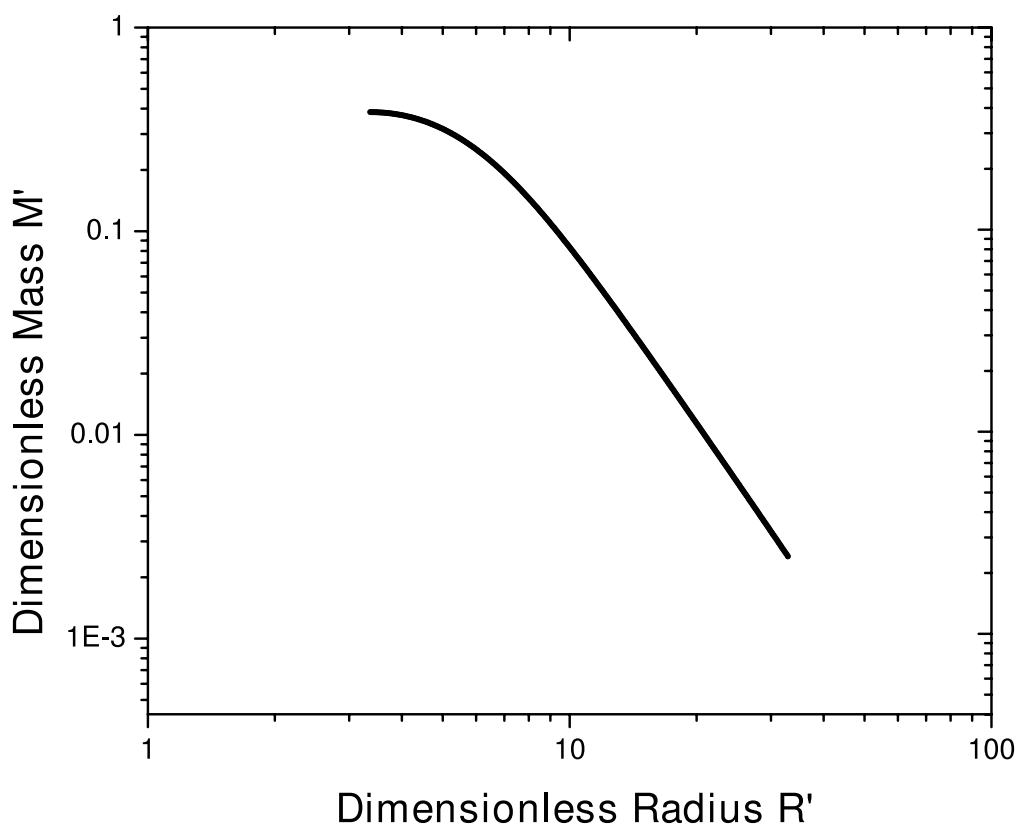

FIG. 3: The above graph shows the $M^{\prime}$ versus $R^{\prime}$ curve on a logarithmic scale for large radii.

star configurations on the right hand side of the maximum in Fig. 2 are stable whereas those on the left hand side are unstable. The maximum in the curve proves the fact that there exists a maximum stable mass for a fermion star, beyond which the stars are unstable and collapse.

Fig. 3 shows $M^{\prime}$ versus $R^{\prime}$ on a double-log scale. For large values of the dimensionless radius $R^{\prime}$, the mass $M^{\prime}$ and the radius $R^{\prime}$ follow the relation

$$
M^{\prime} \cdot R^{\prime \beta}=\text { constant }
$$

A linear fit to the curve for large $R^{\prime}$ gives $\beta=3.0001 \approx 3$ and the constant is $90.97 \approx 91$. Putting these values in the above relation we get

$$
M^{\prime} \cdot R^{\prime 3} \approx 91
$$

The above relation can be also derived analytically by considering a non-relativistic degenerate gas of free fermions and Newtonian gravity. Compact star configurations with a polytrope form of the equation of state, $p \sim \rho^{\gamma}$, are given by the Lane-Emden function [27, 34]. A non-relativistic gas of fermions has a polytrope of $\gamma=5 / 3$ and obeys the Lame-Emden equation of index $(\gamma-1)^{-1}=3 / 2$. For a general polytrope, the relation between $M^{\prime}, R^{\prime}$ and the central density $\rho^{\prime}(0)$ are given by

$$
M^{\prime} \propto \rho^{\prime}(0)^{(3 \gamma-4) / 2} \quad \text { and } \quad R^{\prime} \propto \rho^{\prime}(0)^{(\gamma-2) / 2} \quad \Longrightarrow \quad M^{\prime} \propto R^{(3 \gamma-4) /(\gamma-2)}
$$

and for a polytrope of $\gamma=5 / 3$ one finds $M^{\prime} \propto R^{\prime-3}$, exactly as in eq. (27).

Now we can clearly see the advantages of solving the dimensionless TOV equation. To get full dimensional results for a given fermion mass one should simply rescale the dimensionless mass and radius with factors $a=M_{p}^{3} / m_{f}^{2}$ and $b=M_{p} / m_{f}^{2}$, respectively. For the fermion mass $m_{f}=1 \mathrm{GeV}$, these factors are $a=1.632 M_{\odot}$ and $b=2.410 \mathrm{~km}$. Therefore, for a point on the curve in Fig. 2 with the dimensionless mass $M^{\prime}$ and the dimensionless radius $R^{\prime}$, the actual mass and radius for a fermion star will be given by

$$
M=1.632 M_{\odot} \cdot M^{\prime} \cdot\left(\frac{1 \mathrm{GeV}}{m_{f}}\right)^{2}
$$




\begin{tabular}{cccc}
\hline Fermion mass & $M_{\max }\left(M_{\odot}\right)$ & $R_{\min }$ & comment \\
\hline $100 \mathrm{GeV}$ & $10^{-4}$ & $1 \mathrm{~m}$ & neutralino star (cold dark matter) \\
$1 \mathrm{GeV}$ & 1 & $10 \mathrm{~km}$ & neutron star \\
$1 \mathrm{GeV} / 0.5 \mathrm{MeV}$ & 1 & $10^{3} \mathrm{~km}$ & white dwarf \\
$10 \mathrm{keV}$ & $10^{10}$ & $10^{11} \mathrm{~km}$ & sterile neutrino star \\
$1 \mathrm{keV}$ & $10^{12}$ & $10^{13} \mathrm{~km}$ & axino star (warm dark matter) \\
$1 \mathrm{eV}$ & $10^{18}$ & $10^{19} \mathrm{~km}$ & neutrino star \\
$10^{-2} \mathrm{eV}$ & $10^{22}$ & $10^{23} \mathrm{~km}$ & gravitino star \\
\hline
\end{tabular}

TABLE I: Maximum masses $M_{\max }$ and radii $R_{\min }$ for various cold compact stars made of a free Fermi gas

and

$$
R=2.410 \mathrm{~km} \cdot R^{\prime} \cdot\left(\frac{1 \mathrm{GeV}}{m_{f}}\right)^{2} .
$$

Accordingly, the maximum mass and minimum radius of a fermion star are obtained for $M^{\prime}=0.384$ and $R_{\text {min }}^{\prime}=3.367$,

$$
M_{\max }=0.627 M_{\odot} \cdot\left(\frac{1 \mathrm{GeV}}{m_{f}}\right)^{2}
$$

and

$$
R_{\text {min }}=8.115 \mathrm{~km} \cdot\left(\frac{1 \mathrm{GeV}}{m_{f}}\right)^{2}
$$

One can use the above relations to calculate the masses and radii of different fermion stars. For example, a neutron star with $m_{f}=m_{n}=939.6 \mathrm{MeV}$ in eq. (31) and eq. (32), has the maximum mass $M_{\max }=0.710 M_{\odot}$ at a radius of $R_{\text {min }}=9.192 \mathrm{~km}$, which match very well the original results of Oppenheimer and Volkoff [12].

Next, we consider compact stars built of other fermions utilising the curve shown in Fig. 2. Besides the nucleon and electron, fermions as neutrinos and quarks are well established in the standard model. Moreover, other fermions such as neutralinos, axinos and gravitinos are predicted in supersymmetric extensions of the standard model. For ordinary neutrinos, the mass has been recently constrained to the range from $50 \mathrm{meV}$ to about $1 \mathrm{eV}$ from the measurements of neutrino oscillations and cosmological parameters (see 35, 36]). Hypothetical sterile neutrinos can have typical masses in the keV range (see e.g. 37]). For the supersymmetric particles, neutralinos, gravitinos and axinos, there are large uncertainties concerning their mass ranges. The most likely mass for neutralinos is usually considered to be around $100 \mathrm{GeV}$ but lighter neutralinos with a mass of $\lesssim 50 \mathrm{GeV}$ are discussed in the literature, too [38]. Gravitinos can be heavy or very light in some models, the latter mass range can extend from $10^{-2} \mathrm{eV}$ to $1 \mathrm{keV}$, bounded by constraints from big bang nucleosynthesis and the critical density (see e.g. the discussion in [39]). Axinos, the supersymmetric partner of the axion, were introduced first as a possible warm dark matter candidate with a mass in the keV range 40], but they are discussed now also as a cold dark matter candidate with much higher masses 41]. Compact stars could be also formed from composite dark matter candidates, similar to white dwarfs, e.g. with the heavy charged stable leptons proposed in [42]. Note, that the preceding discussion is just to motivate interesting mass ranges for fermions to be considered in the following. There are of course constraints for dark matter candidates per se which are more severe than the ones mentioned above, see e.g. the recent review on dark matter candidates in [24].

In principle, cold compact stars can be formed out of these exotic fermions, too. Actually, compact stars made of massive neutrinos have been introduced by Markov 43] and calculated within general relativity by Gao and Ruffini [4]. Using the above mentioned masses for these fermions, we have calculated their typical (i.e. maximum) masses and corresponding radii which are listed in Table【 A compact star made of non-interacting neutralinos has a maximum mass of about $10^{-4}$ solar masses with a radius of about one meter. Warm dark matter, fermions with a mass in the $\mathrm{keV}$ range, can form compact objects with galactic masses and a radius of about one light year. In ref. [45, 46] sterile neutrinos with a mass of $m_{f} \sim 50 \mathrm{keV}$ were proposed to explain the dark massive object at the center of our Galaxy, as an alternative to a supermassive black hole. Compact stars made of dark matter interacting with a scalar field have been also considered in [47, 48]. Interestingly, if we take the gravitino mass to be of the order of $10^{-2} \mathrm{eV}^{\mathrm{V}}$, then the corresponding gravitino star has the mass and the radius of our universe. Note, that the above masses and radii are similar to the free-streaming mass and radius scales which are of importance for large-scale structure formation. 


\section{B. Stars made of interacting fermions}

\section{Equation of State for Interacting Fermions}

The previous section was devoted to an idealised case of non-interacting fermions. In a more realistic consideration, the inter-particle interactions must be included, too. In the following, we address the possible impact of interactions on the global properties of fermion stars.

Consider the simplest model of two-body interactions between the fermions. In a lowest order approximation the interaction energy density is proportional to $n^{2}$, where $n$ is the number density of fermions. To have the correct dimensionality this term can be written as $\rho_{\text {int }}=n^{2} / m_{I}^{2}$ where $m_{I}$ represents the energy scale of the interaction. The corresponding contribution to the pressure is:

$$
P_{i n t}=-\left.\frac{\partial E}{\partial V}\right|_{N, T=0}=n^{2} \frac{\left(\partial \rho_{i n t} / n\right)}{\partial n}=\frac{n^{2}}{m_{I}^{2}}
$$

Therefore, in this approximation the energy density and pressure acquire an additional term $n^{2} / m_{I}^{2}$. The interaction must be repulsive so that an increase in the number density increases the pressure and energy density. The scale $m_{I}$ can be also interpreted as the vacuum expectation value of the Higgs field of the interaction. For weak interactions, the interaction strength is just given by Fermi's constant or the vacuum expectation of the Higgs field $v$ generating the masses of the $\mathrm{W}$ and $\mathrm{Z}$ bosons, $G_{F}^{2} / \sqrt{2}=1 /\left(2 v^{2}\right)=(293 \mathrm{GeV})^{-2}$, so that $m_{I} \sim 300 \mathrm{GeV}$. Correspondingly, the strength of low energy strong interactions, quantum chromodynamics (QCD), is controlled by the pion decay constant $1 / f_{\pi}^{2}$ in chiral perturbation theory, with $f_{\pi}=92.4 \mathrm{MeV}$ being the vacuum expectation value of the sigma field (for an introduction to chiral symmetry see e.g. [49]). In quantum hadrodynamics, the expressions for the energy density and pressure are exactly as given above for a repulsive interaction mediated by a vector meson with an interaction strength of $g_{\omega N}^{2} /\left(2 m_{\omega}^{2}\right)$ which is quite close to $1 / f_{\pi}^{2}$ for $g_{\omega N}=13$ and $m_{\omega}=780 \mathrm{MeV}$ (see e.g. [50]). Hence, for strong interactions the typical interaction mass scale is $m_{I} \sim 100 \mathrm{MeV}$. In a more realistic approach, an attractive scalar interaction should be included in addition to a repulsive vector interaction, too. This kind of approach is widely used for baryonic matter [50] as well as for quark stars (see e.g. [51]). In dimensionless variables the energy density and pressure can be written as

$$
\begin{aligned}
\rho^{\prime} \equiv \frac{\rho}{m_{f}^{4}} & =\frac{1}{\pi^{2}} \int_{0}^{z} x^{2} \sqrt{1+x^{2}} d x+\left(\frac{1}{3 \pi^{2}}\right)^{2} y^{2} z^{6} \\
& =\frac{1}{8 \pi^{2}}\left[\left(2 z^{3}+z\right)\left(1+z^{2}\right)^{\frac{1}{2}}-\sinh ^{-1}(z)\right]+\left(\frac{1}{3 \pi^{2}}\right)^{2} y^{2} z^{6} \\
p^{\prime} \equiv \frac{p}{m_{f}^{4}} & =\frac{1}{3 \pi^{2}} \int_{0}^{z} \frac{x^{4}}{\sqrt{1+x^{2}}} d x+\left(\frac{1}{3 \pi^{2}}\right)^{2} y^{2} z^{6} \\
& =\frac{1}{24 \pi^{2}}\left[\left(2 z^{3}-3 z\right)\left(1+z^{2}\right)^{\frac{1}{2}}+3 \sinh ^{-1}(z)\right]+\left(\frac{1}{3 \pi^{2}}\right)^{2} y^{2} z^{6}
\end{aligned}
$$

where $z$ is the dimensionless Fermi momentum and $y=m_{f} / m_{I}$ the interaction strength.

What are the values to be taken for the interaction strength $y$ ? For a realistic neutron star, neutrons interact strongly with $m_{I} \sim 100 \mathrm{MeV}$, as outlined above. With a neutron mass of $m_{f} \sim 1 \mathrm{GeV}$ one arrives at $y \sim 10$. Neutrinos interact weakly and with a neutrino mass of about $1 \mathrm{eV}$ (for sterile neutrinos $m_{f} \sim 1 \mathrm{keV}$ ), one finds $y \sim 10^{-11}$ (for sterile neutrinos $y \sim 10^{-8}$ ). For neutralinos with a mass of $100 \mathrm{GeV}$ and weak interactions, $y \sim 1 / 3$, for strongly interacting neutralinos $y \sim 10^{3}$. We find that for small values of $y$ the mass-radius relation remains almost unchanged because the equation of state is dominated by the kinetic terms. Any change in the equation of state can only occur for $y \geq 1$, i.e. when interaction term starts dominating the equation of state before the fermions are becoming relativistic. Hence, it is sufficient to take $y$ to be in the range $10^{-2}$ to $10^{3}$. Below $y=10^{-2}$ one will hardly observe any change in the mass-radius relation.

From the equations for $p$ and $\rho$ one finds that the common factor $m_{f}^{4}$ can be taken out, so that general scaling arguments result in $\epsilon_{\circ}=m_{f}^{4}$. Therefore, the dimensionless forms of $p$ and $\rho$ are $p^{\prime}=p / m_{f}^{4}$ and $\rho^{\prime}=\rho / m_{f}^{4}$ and the corresponding mass $M$ and radius $R$ are $M^{\prime}=M / a$ and $R^{\prime}=R^{\prime} / b$ with $a=M_{p}^{3} / m_{f}^{2}$ and $b=M_{p} / m_{f}^{2}$, exactly Landau's mass and radius as before.

Note, that for each value of $y$ there is a different equation of state and two different regimes exist: $z \ll 1$, the non-relativistic limit, and $z \gg 1$, the relativistic limit. For small $y \ll 1$, the equation of state will be that of an 


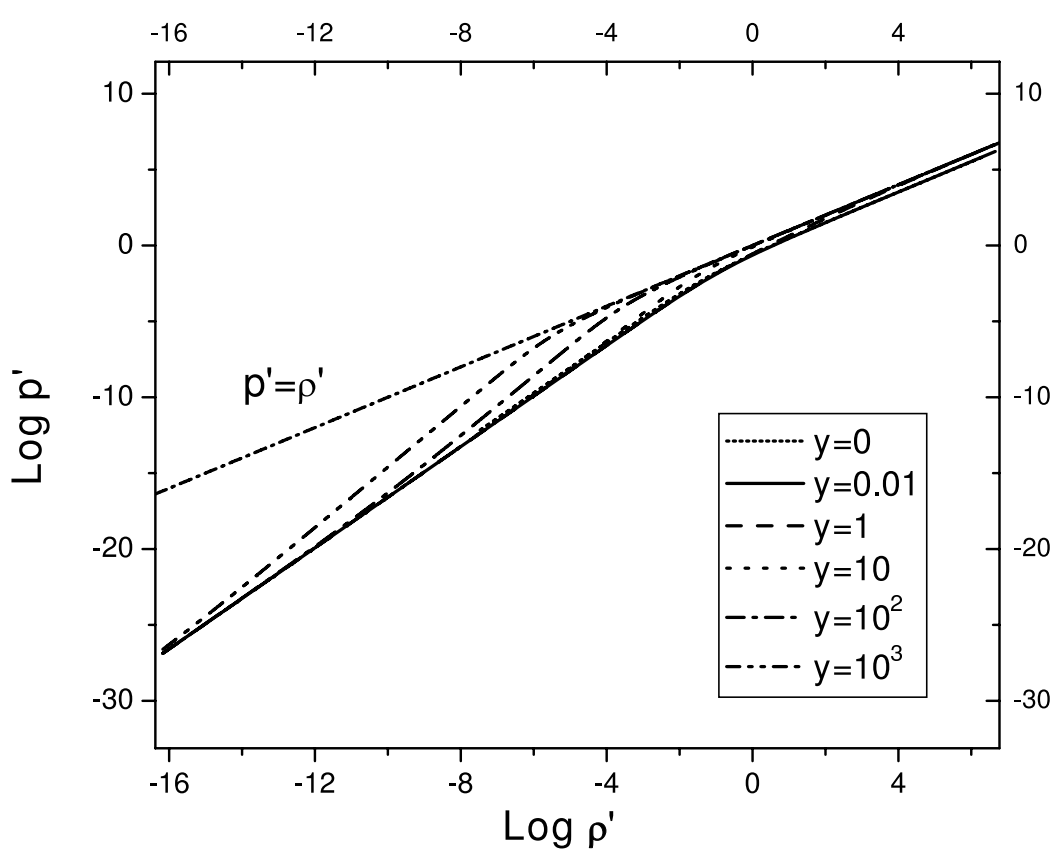

FIG. 4: The equation of state in dimensionless form, $\log p^{\prime}$ versus $\log \rho^{\prime}$, for different values of the interaction strength as indicated in the figure.

ideal Fermi gas and for large $y \gg 1$ the equation of state will be mostly determined by the interaction term, unless $z$ becomes small enough so that the ideal gas term becomes the dominant ones.

The dimensionless TOV equations (14) and (15) are solved by making use of the dimensionless equation of state, eqs. (34) and (35). The equation of state depends on two parameters, $z$ and $y$. To solve the dimensionless TOV equations for particular values of $y$, we first construct the dimensionless equation of state in a tabular form for different values of $z$. Linear interpolation is used to find $p^{\prime}$ corresponding to a particular dimensionless density $\rho^{\prime}$ and vice-versa. As before, the dimensionless TOV eq. (14) and eq. (15) are numerically solved using a fourth order Runge-Kutta algorithm. To plot the graph of $M^{\prime}$ versus $R^{\prime}$ for various $y$, we take 100 equally spaced values of $y$ lying between $10^{-2}$ and $10^{3}$. For each value of $y$, the $M^{\prime}$ versus $R^{\prime}$ curve is computed for 50 equally spaced dimensionless central densities. The program adjusts the step size in the dimensionless radius to have about 2000 points for each

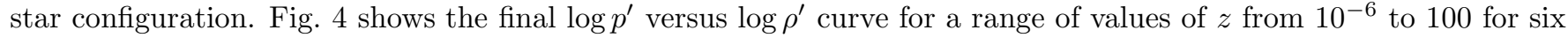
different values of $y$.

From Fig. 4 one makes the following observations:

1. For large values of $\rho^{\prime}$, the different equations of state merge to one line, except for the curve of the cases $y=0$ and $y=0.01$ which stay slightly below the other ones.

2. The transition point from the relativistic curve to a steeper one shifts to lower densities for increasing interaction strength $y$.

3. For very small values of $\rho^{\prime}$, the slope of the curve is approaching the slope for a non-interacting ideal gas. The higher the interaction strength $y$, the lower $\rho^{\prime}$ must be taken to reach the non-interacting limit.

These observations can be explained in the following manner. The equations (34) and (35) are parametric in $z$ and $y$. In the non-relativistic limit, $z \ll 1$, they are reduced to

$$
\rho^{\prime} \approx \frac{z^{3}}{3 \pi^{2}}+\left(\frac{1}{3 \pi^{2}}\right)^{2} y^{2} z^{6} \quad(z \ll 1)
$$

and

$$
p^{\prime} \approx \frac{z^{5}}{15 \pi^{2}}+\left(\frac{1}{3 \pi^{2}}\right)^{2} y^{2} z^{6} \quad(z \ll 1)
$$


In the relativistic limit, $z \gg 1$, one finds

$$
\rho^{\prime} \approx \frac{z^{4}}{4 \pi^{2}}+\left(\frac{1}{3 \pi^{2}}\right)^{2} y^{2} z^{6} \quad(z \gg 1)
$$

and

$$
p^{\prime} \approx \frac{z^{4}}{12 \pi^{2}}+\left(\frac{1}{3 \pi^{2}}\right)^{2} y^{2} z^{6} \quad(z \gg 1)
$$

If the interaction strength is small, $y \ll 1$, the interaction terms in eqs. (36) and (37) can be ignored for $z \ll 1$ and one recovers for small densities the non-interacting case. For increasing $y$, the interaction terms become more and more important. Let us examine the ratio of the interaction term to the free gas term in more detail for the different cases. We define the two ratios $\tau_{\rho}(z \ll 1)$ and $\tau_{p}(z \ll 1)$ for the dimensionless energy density and pressure eqs. (36) and (37) as

$$
\tau_{\rho}(z \ll 1) \sim y^{2} z^{3} \quad \text { and } \quad \tau_{p}(z \ll 1) \sim y^{2} z
$$

The corresponding ratios $\tau_{\rho}(z \gg 1)$ and $\tau_{p}(z \gg 1)$ for the case $z \gg 1$ follow from eqs. (38) and (39),

$$
\tau_{\rho}(z \gg 1) \sim y^{2} z^{2} \quad \text { and } \quad \tau_{p}(z \gg 1) \sim y^{2} z^{2}
$$

For non-relativistic fermions, $z \ll 1$, assume first that the interaction strength $y$ is such that $\tau_{\rho} \ll 1$ and $\tau_{p} \ll 1$. Then the interaction terms can be ignored and one recovers the standard equation of state for a free non-relativistic gas, a polytrope of $\gamma=5 / 3$. For larger values of the interaction strength, the interaction terms become important already in the non-relativistic regime, more drastically for the pressure, eq. (37), than for the energy density, eq. (36), as for the same value of $y, \tau_{\rho} \sim z^{3}$ while $\tau_{p} \sim z$. Hence, the curve for the interacting case will be above the one for the non-interacting gas, an effect which increases with increasing interaction strength $y$. In the region where $\tau_{\rho} \gg 1$ and $\tau_{p} \gg 1$, the interaction terms dominate and the equation of state reduces to $p^{\prime}=\rho^{\prime}$, which is the stiffest equation of state consistent with special relativity. This equation of state was first considered by Zeldovich [52] (see the discussion in [53]). Note, that this condition is fulfilled already for $z \gg y^{-2 / 3}$, which for large values of $y$ is substantially smaller than one. Therefore, even for non-relativistic fermions one can reach the causal limit, where $p^{\prime}=\rho^{\prime}$, for an strongly interacting gas at $\rho^{\prime} \ll 1$.

For relativistic fermions, $z \gg 1$, consider first the interaction strength $y$ to be such that $\tau_{\rho} \gg 1$ and $\tau_{p} \gg 1$. Then the free gas terms in eqs. (38) and (39) can be ignored and the equation of state reduces simply to $p^{\prime}=\rho^{\prime}$. Thus, both in the relativistic and in the non-relativistic limit, the equation of state will have the form $p^{\prime}=\rho^{\prime}$. For small values of $y$, a different regime for the equation of state is reached when $\tau_{\rho} \ll 1$ and $\tau_{p} \ll 1$. In that case the equation of state becomes the one of an ultra-relativistic gas $p^{\prime}=\rho^{\prime} / 3$, see eq. (17). This happens for e.g. $y=0.01$ and $z \sim 10$ in Fig. 4

With the above arguments we are now in the position to explain the behaviour of the curves in Fig. 4 . For $y=0.01$ and for relativistic fermions, the equation of state is that for an ultra-relativistic free gas, $p^{\prime}=\rho^{\prime} / 3$, eq. (17) which differs from $p^{\prime}=\rho^{\prime}$, the one for an interaction-dominated Fermi gas with large values of the interaction strength $y \gg 1$. The different prefactor explains the slight difference between the lines for $y=0.01$ and the other curves with larger values of $y$ for large densities $\rho^{\prime}$. There appears a sharp change of the slope of the curves which signals the transition from the energy density being dominated by interactions to being dominated by the (free) kinetic terms. For even smaller densities $\rho^{\prime} \ll 1$, the equations of state are eventually given by the non-relativistic polytrope of eq. (16). Deviations from the non-interacting case arise for intermediate densities with increasing interaction strength $y$, since the interaction increases the pressure more rapidly than the energy density.

\section{Solution to the TOV equation for interacting fermions}

We solve the dimensionless TOV, eqs. (14) and (15), with the dimensionless pressure and energy density as given by eqs. (34) and (35). The equation of state depends now on the interaction strength parameter $y$. The plot of the dimensionless mass $M^{\prime}$ versus radius $R^{\prime}$ for different values of $y$ is depicted in Fig. 5 on a double-log scale.

From Fig. 5 we observe that:

1. For small interactions strengths $y \ll 1$, the mass-radius curves are very close to each other. The interaction terms can be neglected in this case and the equation of state is determined by the free gas term, which results in nearly the same mass-radius curves. 


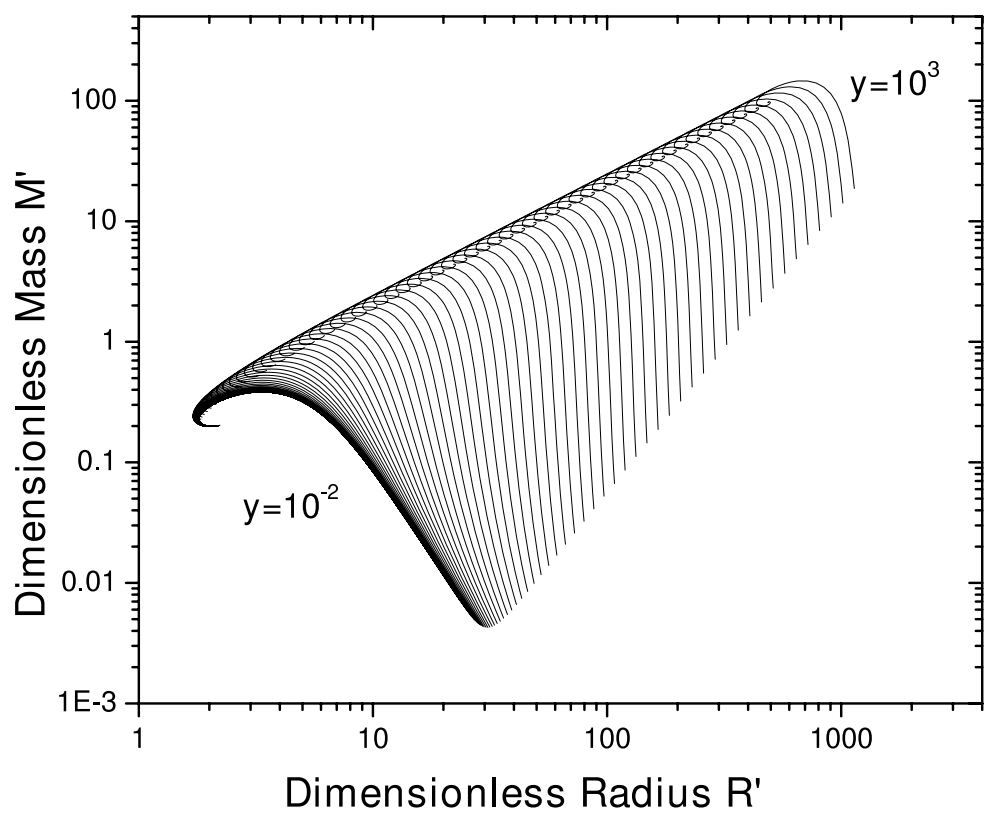

FIG. 5: Mass-radius relation in dimensionless units for different values of the interaction strength $y$ in the range $10^{-2}($ lowest curve) to $10^{3}$ (highest curve) in 100 equal steps.

2. For large interaction strengths, $y \geq 1$, the mass-radius curves are shifted towards larger masses and radii. As the interaction becomes stronger, the equation of state is getting more and more stiff approaching $p^{\prime}=\rho^{\prime}$.

3. The mass-radius curves, in particular the maximum mass and the corresponding radius, seem to follow a common trend for strongly interacting fermions, so a scaling behaviour should be expected.

4. The slope of the mass-radius curve for masses well below the maximum mass changes with increasing interaction strength $y$ from the free case behaviour $M^{\prime} \times{R^{\prime}}^{3}=$ const. to one with a rather constant radius for a wide range of masses.

In order to obtain the dimensionful mass and radius for different values of $y$ one should use eqs. (29) and (30) and take the numerical values for $M^{\prime}$ and $R^{\prime}$ from the corresponding curve in Fig. 5 . For a neutron star, strong interactions between neutrons mediated by vector mesons sets the typical interaction strength to be around $y=10$. Then, the maximum stable mass emerges to be $3.77 M_{\odot}$ instead of $0.71 M_{\odot}$ as was found earlier for non-interacting neutrons. Note, that attractive forces will reduce this value somewhat closer to the maximum masses considered presently for realistic equations of state (see e.g. [54]). For an interacting neutralino star, weak interactions result in low values for the interaction strength, $y \sim 1 / 3$, so that the maximum mass would stay unchanged. However, if we assume strong interaction values for $y$ as motivated by $\mathrm{QCD}$ of around $y=10^{3}$, the maximum stable mass is boosted to $2.7 \times 10^{-2} M_{\odot}$ compared to $6.3 \times 10^{-5} M_{\odot}$ for the non-interacting case.

Next we discuss the behaviour of the mass-radius relation for large values of $R^{\prime}$ considering strongly interacting fermions, i.e. $y \gg 1$. The question is, is there any relation similar to the one for the non-interacting case, eq. (27)? Note first, that the tail of the $M^{\prime}$ versus $R^{\prime}$ curve corresponds to small central densities of the star $\rho^{\prime}(0)$, so that the dimensionless Fermi momentum $z=k_{f} / m_{f}$ is quite small, i.e. $z \ll 1$. In the non-relativistic regime, it is likely that the equation of state can be described by a polytrope, even for interacting fermions.

Indeed, at $z \ll 1$, the equation of state reduces to eqs. (36) and (37) and one can ignore the interaction term proportional to $z^{6}$ as compared to the term proportional to $z^{3}$. Thus one finds that

$$
\rho^{\prime} \simeq \frac{z^{3}}{3 \pi^{2}}
$$


Now, using this equation to eliminate $z$ from eq. (37), we get

$$
p^{\prime} \simeq \frac{\left(3 \pi^{2}\right)^{2 / 3}}{5} \rho^{\prime 5 / 3}+y^{2} \rho^{\prime 2}
$$

For large values of $y \gg 1$, the interaction term becomes important for the pressure above a certain Fermi momentum, when $\tau_{p}(z \ll 1) \sim 1$ or $z \sim 1 / y^{2}$. Then, the pressure grows with the square of the energy density and the corresponding polytropic coefficient is $\gamma=2$. Using the Lane-Emden solution, (28), one finds that the radius does not depend on the central density, while the mass increases linearly with the central density, hence $R^{\prime}=$ const. for a large range of masses. Examining Fig. 4 we see that this behaviour is present for $y \gg 1$ close to the point where the causal limit, $p^{\prime}=\rho^{\prime}$, is reached. The upper limit to the energy density is therefore given by the point where the interaction starts to affect the energy density in addition to the pressure, i.e. $\tau_{\rho}(z \ll 1) \sim 1$ or $\rho \sim z^{3} \sim y^{-2}$. The critical lower value is given as discussed above by the condition $\tau_{p}(z \ll 1) \sim 1$, i.e. $z \sim y^{-2}$ or $\rho^{\prime} \propto y^{-6}$, where the interaction starts to affect the pressure. Therefore, radii are constant for the mass range extending from $M^{\prime} \propto \rho^{\prime} \propto y^{-6}$ to $M^{\prime} \propto \rho^{\prime} \propto y^{-2}$, which can reach, for the case $y=10^{3}$, up to about twelve orders of magnitude in compact star masses!

\section{Scaling behaviour for interacting fermions}

As mentioned above, a scaling behaviour is expected for large values of $y$. Consider eq. (36) and eq. (37) for nonrelativistic fermions and for $y \gg 1$. Then the dimensionless pressure $p^{\prime}$ is dominated by the interaction term while the dimensionless energy density $\rho^{\prime},(36)$, contains both the kinetic and interaction terms. One can see that in this case the equation of state will turn from the non-relativistic polytrope, (16), to the causal limit of the form $p^{\prime}=\rho^{\prime}$ when the fermions are still non-relativistic, i.e. $z \ll 1$. Indeed, one can write the dimensionless energy density as:

$$
\begin{aligned}
\rho^{\prime} & =\frac{z^{3}}{3 \pi^{2}}+\left(\frac{1}{3 \pi^{2}}\right)^{2} y^{2} z^{6} \\
& =\frac{z^{3}}{3 \pi^{2}}+p^{\prime}
\end{aligned}
$$

Hence, the pressure $p^{\prime}$ is larger than the kinetic term of the energy density for $z^{3} \sim y^{2} z^{6}$ or $z^{3} \sim 1 / y^{2}$. In this regime, both $p^{\prime}$ and $\rho^{\prime}$ are of the order of $\sim 1 / y^{2}$. Hence, the pressure and energy density can be rescaled in a dimensionless form with the factor $m_{f}^{4} / y^{2}$. The corresponding Landau mass and Landau radius will be modified accordingly to

$$
M_{L}^{i n t}=M_{p}^{3} / m_{f}^{2} \cdot y \quad \text { and } \quad R_{L}^{i n t}=M_{p} / m_{f}^{2} \cdot y
$$

respectively, so that the maximum mass and the corresponding radius increase linearly with the interaction strength $y$. We note in passing that the importance of the interaction on the global properties of compact objects has been also noted for the case of boson stars with interacting scalar fields in [55].

The numerical results for the scaling behaviour of the maximum mass as a function of the interaction strength $y$ are plotted in Fig. 6] on a double-log scale. For small values of $y$, the maximum mass $M_{\max }^{\prime}$ does not change and basically remains constant. For $y \sim 1$, the maximum mass $M_{\text {max }}^{\prime}$ starts increasing as a function of $y$, approaching a power-law rise for $y \gg 1$.

For small values of $y$, the interactions do not affect significantly the equation of state and there will be almost no change in the maximum mass. If the interaction terms in the equation of state become comparable to the ones of the free gas, i.e. if $y \sim 1$, the maximum mass begins to increase. Finally, for $y \gg 1$, the pressure and the energy density for the maximum mass configuration are dominated by the interaction terms, which can be scaled out by the factor $1 / y^{2}$ so that the maximum mass will increase linearly with $y$ as explained above. For the part of the graph in Fig. 6 where $y \gg 1$, one can perform the following general fit:

$$
M_{\max }^{\prime}=c_{1}+s_{1} \cdot y^{\gamma_{1}}
$$

as $\log M_{\text {max }}^{\prime}$ is linear in $\log y$ for $y \gg 1$. The constant $c_{1}$ is fixed by the numerical result found for the non-interacting case, hence $c=0.384$. The parameters $s_{1}$ and $\gamma_{1}$ are fitted to the curve of Fig. 6 to be $s_{1}=0.165$ and $\gamma_{1}=0.999 \approx 1$. The maximum mass $M_{\max }$ of a compact star can then be calculated from the relation

$$
M_{\max }=(0.384+0.165 \cdot y) \cdot\left(\frac{1 \mathrm{GeV}}{m_{f}}\right)^{2} \cdot 1.632 M_{\odot}
$$

as an approximation to our numerical solution of the full TOV equations. 


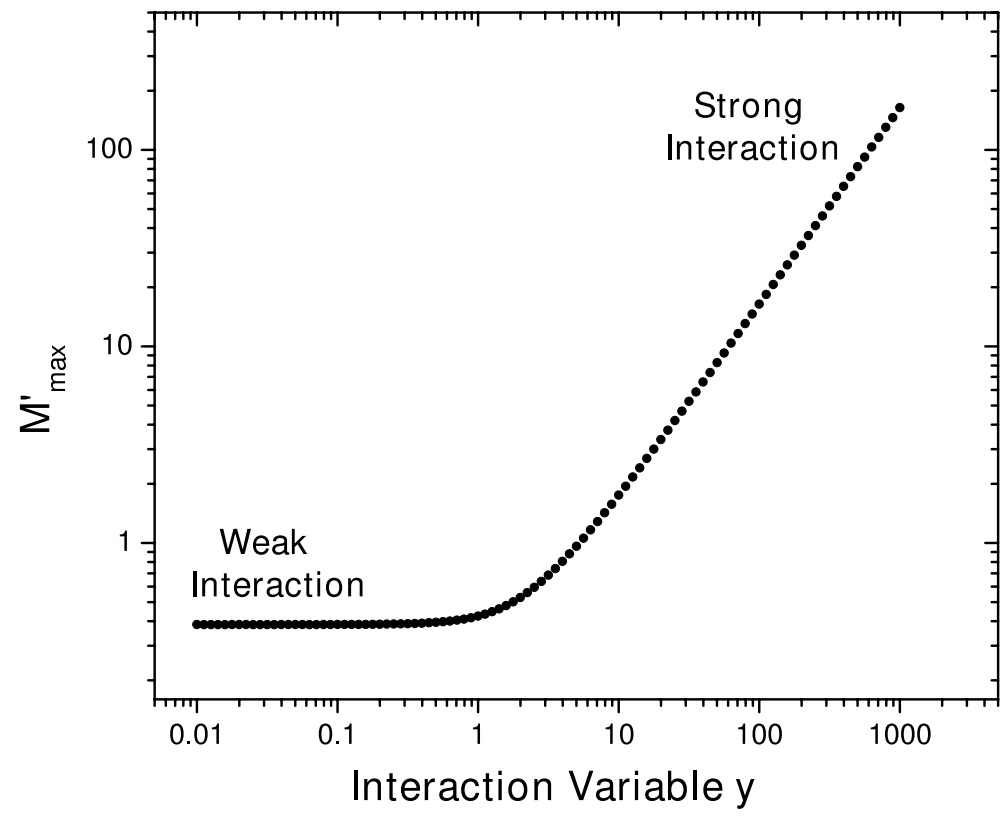

FIG. 6: The dimensionless maximum mass $M_{\text {max }}^{\prime}$ for interacting fermions versus the interaction strength $y$ on a double-log scale. For small values of $y$, the maximum mass does not change, while it increases with a power law for strong interactions $(y>1)$.

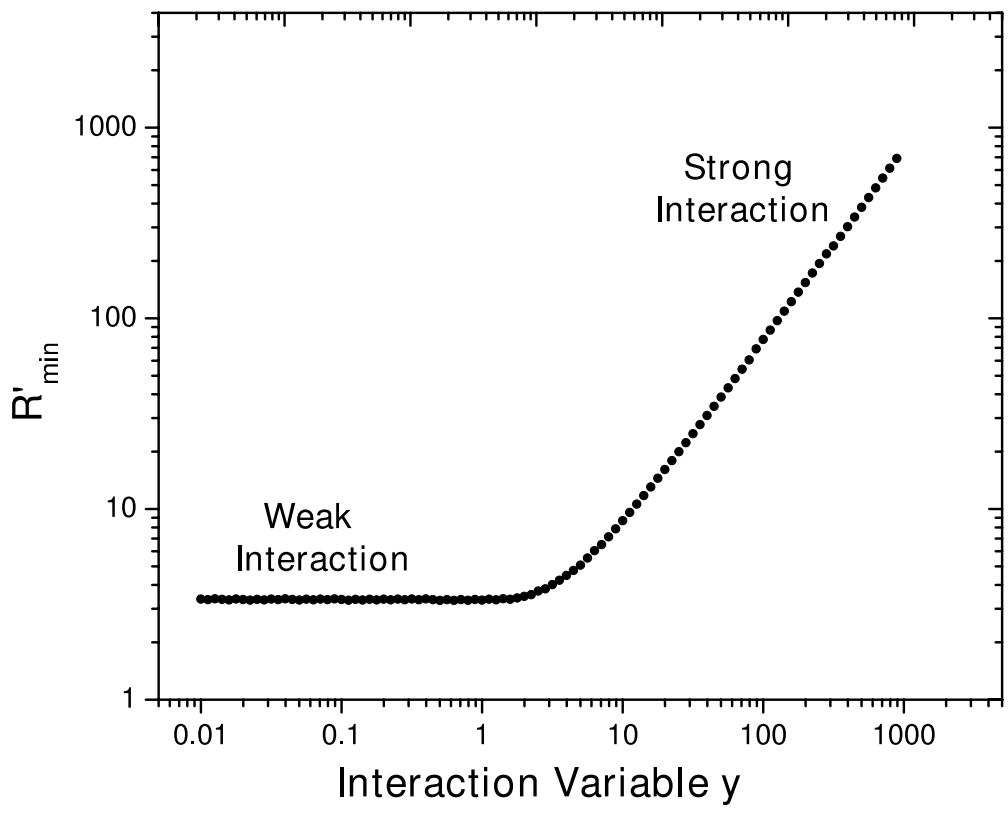

FIG. 7: The minimum dimensionless radius $R_{m i n}^{\prime}$ for the maximum mass configurations as a function of the interaction strength $y$ on a double-log scale. 


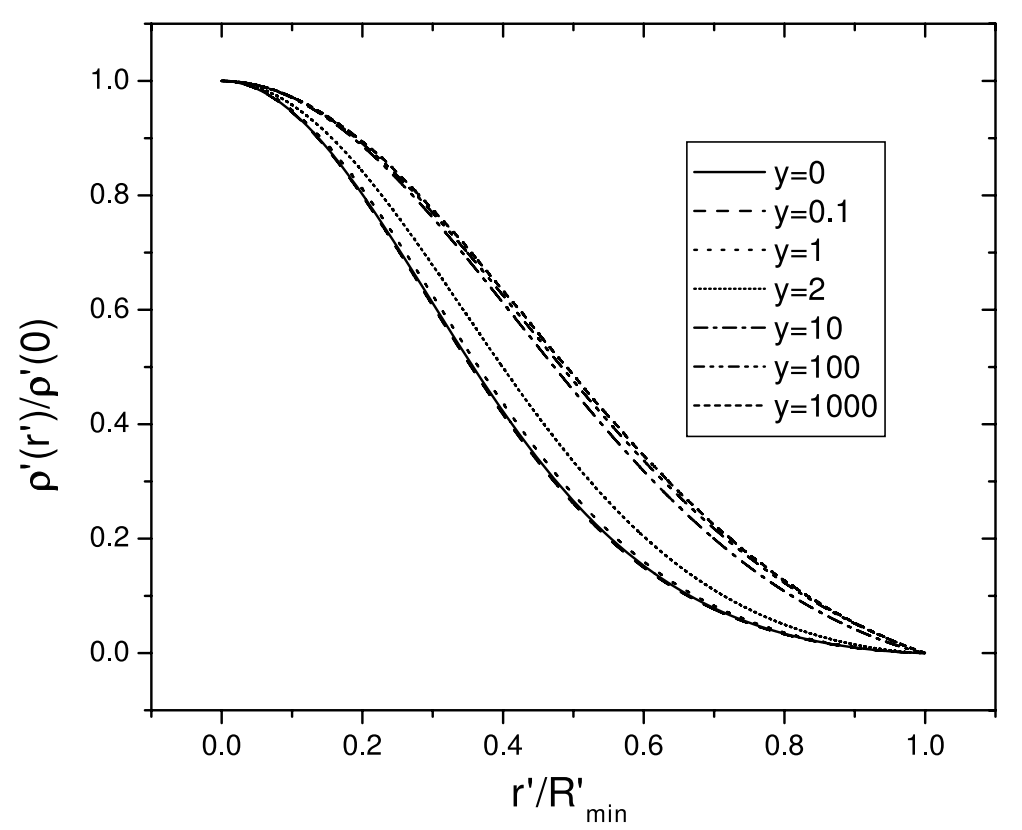

FIG. 8: The energy density $\rho^{\prime}\left(r^{\prime}\right)$ versus the radius $r^{\prime}$, for star configurations with different values of the interaction strength $y$.

We plot the minimum radius $R_{\min }^{\prime}$ versus $y$ on a double-log scale in order to extract a scaling behaviour for the radius in Fig. [7. For small values of $y$, the radius $R_{\min }^{\prime}$ stays constant. For $y>1$, the radius increases with the interaction strength following again a power-law behaviour, so that we take the following fitting expression for $R_{\text {min }}^{\prime}$ :

$$
R_{\min }^{\prime}=c_{2}+s_{2} \cdot y^{\gamma_{2}}
$$

where $c_{2}, s_{2}$ and $\gamma_{2}$ are constants. The constant $c_{2}$ is fixed by the non-interacting limit as discussed before, i.e. by $R_{\min }^{\prime}$ for a free Fermi gas, for which numerically $c_{2}=3.367$. In the $y \gg 1$ limit, one obtains $s_{2}=0.797$ and $\gamma_{2}=0.9942 \sim 1$. Hence, for large interactions strengths $y \gg 1$ the radius can be approximated as

$$
R_{\text {min }}=(3.367+0.797 \cdot y) \cdot\left(\frac{1 \mathrm{GeV}}{m_{f}}\right)^{2} \cdot 2.410 \mathrm{~km}
$$

According to the scaling arguments as derived above, the minimum radius (as well as the maximum mass) should increase linearly with $y$ for large interaction strengths which is indeed being found numerically and observed in Fig. [6 for the maximum mass and in Fig. 7 for the corresponding radius. For small values of $y$, scaling arguments predict a nearly constant minimum radius as a function of $y$ as also clearly seen in Fig. [6 and in Fig. [7]

However, one should expect a difference in the radial density profile for the weak and the strong interaction cases. When interactions are weak, the equation of state is governed by the ideal Fermi gas terms in the core of the compact star. For increasing interaction strength, the pressure increases more rapidly due to the interaction terms for low energy densities. As a result, at the surface of the compact star, the energy density decreases more rapidly and the additional pressure from the interactions becomes important. A halo is created as more matter can be supported against the gravitational pull. The total mass of the star remains almost the same as it is mainly given by the dense core and not affected by the dilute halo. Interestingly, also the corresponding radius is not modified substantially by this effect. This behaviour is illustrated in Fig. 8 which shows the density profile for various star configurations with different values of the interaction strength $y$. The dimensionless density $\rho^{\prime}\left(r^{\prime}\right)$ is plotted versus the dimensionless radius of the star $r^{\prime}$ for several configurations. Note, that the curves are drawn for the maximum mass configurations and normalised to their central density and the total radius. Fig. 8 shows the presence of a dense core and a low density halo at the surface of the star. The rate of the decrease of $\rho^{\prime}\left(r^{\prime}\right)$ with the radius $r^{\prime}$ is large for small values of $y$ and decreases gradually for higher values of $y$ generating a denser halo of the compact star for strongly interacting fermions compared to the weakly or non-interacting ones. 


\section{SUMMARY AND DISCUSSIONS}

We construct general equations of state for fermions of arbitrary mass $m_{f}$ to be used as input for solving the TOV equations for corresponding compact stars. Besides a free gas of fermions, we consider also the case of interacting fermions by adding interaction terms $\sim n^{2}$ to the energy density and pressure. We discuss various ways of rescaling the TOV equation and find the corresponding scaling solutions for compact star configurations for arbitrary fermion mass and interaction strength. The scaling solutions are tested by detailed numerical calculations.

In particular, we have demonstrated that Landau's argument and the corresponding expressions for the maximum mass and the corresponding (minimum) radius hold also in the case of general relativity. For a compact star made of fermions, there exists an upper limit for the mass which is of the order of the Landau mass $M_{p}^{3} / m_{f}^{2}$, where $M_{p}$ is the Planck mass. The corresponding radius scales as the Landau radius $M_{p} / m_{f}^{2}$. By directly solving the TOV equations, we have found the mass-radius relation in dimensionless form, i.e. in units of the Landau mass and Landau radius, supporting our analytic finding. The numerical values of the maximum mass and the corresponding radius are $M_{\max }=0.384 M_{p}^{3} / m_{f}^{2}$ and $R_{\text {min }}=3.367 M_{p} / m_{f}^{2}$, respectively. For large radii the mass and the radius are related by the well-known relation $M \cdot R^{3}=$ const. The results are in accordance with those known for a free neutron gas but our scaling relations can be used for fermions of any mass. For example, fermions with a mass of $100 \mathrm{GeV}$ can form compact objects up to a maximum mass of about $10^{-4} M_{\odot}$ and radii down to 1 meter as deduced from the same mass-radius relation as for a free gas of neutrons, when properly rescaled by the Landau mass and Landau radius. The same can be done for the mass-radius relation of neutrino stars. For $m_{f} \simeq 1 \mathrm{eV}$ the corresponding maximum mass and its radius are, however, of the order of $M_{\max } \sim 10^{18} M_{\odot}$ and $R_{\min } \sim 10^{19} \mathrm{~km}$. Interestingly, a hypothetical fermion with a mass of about $10^{-2} \mathrm{eV}$ can have a maximum mass and a corresponding radius which matches the total mass and the horizon of the present universe.

Effects from interactions between the fermions are taken into account by adding terms proportional to the fermion density squared to the expressions for the pressure and energy density. This can be motivated by e.g. such effective models of strong interactions as quantum hadrodynamics. The resulting equation of state depends on a new variable $y=m_{f} / m_{I}$ which controls the strength of the interaction. The mass $m_{I}$ fixes the range and strength of the interactions. The values of $y$ can be as small as $10^{-11}$ for neutrinos with a mass of $\sim 1 \mathrm{eV}$ and as high as $y=10^{3}$ for strongly interacting neutralinos with a mass of $\sim 100 \mathrm{GeV}$.

Using scaling arguments we arrive at the dimensionless equation of state and corresponding scaling solutions for the maximum mass and its radius for interacting fermions. We show that the maximum mass and the radius are rather constant for small interaction strengths, $y \ll 1$, but are entirely determined by the interaction terms for $y \gg 1$ : Both, the maximum mass and its radius increase linearly with the interaction strength for $y \gg 1$. The scaling is supported by numerical calculations, where we find that $M_{\max } \approx 0.165 y \cdot M_{p}^{3} / m_{f}^{2}$ and $R_{\max } \approx 0.797 y \cdot M_{p} / m_{f}^{2}$ for $y \gg 1$. Besides these general scaling features, there is a small change in the density profile for large interaction strengths, which can be attributed to the formation of an enhanced halo density in the outer region of the star. For large interaction strengths the mass-radius relation changes from the standard $M \cdot R^{3}=$ const behaviour to the one with a constant radius for a wide range of compact star masses. This is because the pressure increases as the square of the energy density and not as the power $5 / 3$ for a free non-relativistic Fermi gas. The mass range for constant radii changes with the interaction strength in the range between $y^{-6}$ and $y^{-2}$. Specifically, for values of the interaction strength of $y=10^{3}$ and a fermion mass of $100 \mathrm{GeV}$, the maximum mass increases from the value for the non-interacting case, $M_{\max } \sim 10^{-4} M_{\odot}$ to $M_{\max } \sim 10^{-1} M_{\odot}$, which is comparable to the one for ordinary neutron stars. The compact star mass range, where the radius stays constant, extends from about the maximum mass of about $10^{-1} M_{\odot}$ down to $10^{-13} M_{\odot}$ with a typical radius as given by the Landau radius, i.e. about 100 meter.

There are two important issues which require a special study which is beyond the scope of the present paper.

First, the crucial assumptions for all these investigations is that the fermions are stable on the time scale comparable with the lifetime of the universe, i.e. $\tau \geq H_{0}^{-1} \approx 14 \mathrm{Gyr}$, where $H_{0}$ is the present value of the Hubble constant. In other words, it is assumed that the fermions constituting the compact star are conserved, i.e. there is no annihilation into other kinds of matter. Naive estimates for the lifetime using $\tau \sim(n \cdot \sigma)^{-1}$, with the number density $n \sim m_{f}^{3}$ and the cross section $\sigma \sim m_{f}^{2} / m_{I}^{4}$, result in the constraint $m_{f}<\left(H_{0} \cdot m_{I}^{4}\right)^{1 / 5}$. For weak interactions, one arrives at fermion masses $m_{f}<1 \mathrm{keV}$, for gravitational interactions at the Planck scale $m_{I}=M_{p}$, however, the fermion mass must be only lower than $m_{f}<10^{4} \mathrm{TeV}$ (in all cases $y \ll 1$ ).

The second important question is, when and how the compact objects made of exotic fermions could be formed? One may speculate that this could happen at very early stages in the history of the universe, right after the inflation stage. These early formed objects could serve as seeds for clumping ordinary matter at later stages of the expansion, after the radiation decoupling. Therefore, one may speculate about hybrid objects where exotic fermion stars are surrounded by the halos of ordinary matter.

Our final remark concerns the possible observations of compact objects made of dark matter particles. In fact, 
there exist limits derived from the observation of micro-lensing events. The MACHO collaboration has excluded the mass range of $\left(10^{-7}-30\right) M_{\odot}$ for compact objects forming the bulk of the Galactic dark matter. However, compact stars of these mass ranges are not ruled out if they do not contribute more than $4 \cdot 10^{11} M_{\odot}$ to the Galactic halo (see [56] and references therein).

\section{Acknowledgments}

This work has started as a summer research project of Gaurav Narain at the Goethe University in Frankfurt, Germany. GN thanks the institute for theoretical physics for their warm hospitality. JSB thanks Stefan Hofmann for several discussions on dark mater and dark stars. This work is supported in part by the Gesellschaft für Schwerionenforschung (Germany), and the grants RFBR 05-0204013 and NS-8756.2006.2 (Russia).

[1] N. K. Glendenning, Compact Stars - Nuclear Physics, Particle Physics, and General Relativity (Springer, New York, 2000), 2nd ed.

[2] F. Weber, Pulsars as Astrophysical Laboratories for Nuclear and Particle Physics (Institute of Physics, Bristol, 1999).

[3] S. L. Shapiro and S. A. Teukolsky, Black Holes, White Dwarfs, and Neutron Stars: The Physics of Compact Objects (John Wiley \& Sons, New York, 1983).

[4] R. R. Silbar and S. Reddy, Am. J. Phys. 72, 892 (2004), nucl-th/0309041.

[5] R. Balian and J.-P. Blaizot, Am. J. Phys. 67, 1189 (1999), cond-mat/9909291.

[6] C. B. Jackson, J. Taruna, S. L. Pouliot, B. W. Ellison, D. D. Lee, and J. Piekarewicz, Eur. J. Phys. 26, 695 (2005), astro-ph/0409348.

[7] J. Macher and J. Schaffner-Bielich, Eur. J. Phys. 26, 341 (2005), astro-ph/0411295.

[8] I. Sagert, M. Hempel, C. Greiner, and J. Schaffner-Bielich (2005), astro-ph/0506417.

[9] R. H. Fowler, Mon. Not. R. Astron. Soc. 87, 114 (1926).

[10] S. Chandrasekhar, Astrophys. J. 74, 81 (1931).

[11] L. D. Landau, Physik. Zeits. Sowjetunion 1, 285 (1932).

[12] J. R. Oppenheimer and G. M. Volkoff, Phys. Rev. 55, 374 (1939).

[13] V. A. Ambartsumyan and G. S. Saakyan, Sov. Astron. 4, 187 (1960).

[14] N. K. Glendenning, Astrophys. J. 293, 470 (1985).

[15] P. J. Ellis, R. Knorren, and M. Prakash, Phys. Lett. B 349, 11 (1995).

[16] R. Knorren, M. Prakash, and P. J. Ellis, Phys. Rev. C 52, 3470 (1995).

[17] J. Schaffner and I. N. Mishustin, Phys. Rev. C 53, 1416 (1996).

[18] D. D. Ivanenko and D. F. Kurdgelaidze, Astrophys. 1, 251 (1965).

[19] N. Itoh, Prog. Theor. Phys. 44, 291 (1970).

[20] E. Witten, Phys. Rev. D 30, 272 (1984).

[21] P. Haensel, J. L. Zdunik, and R. Schaeffer, Astron. Astrophys. 160, 121 (1986).

[22] C. Alcock, E. Farhi, and A. Olinto, Astrophys. J. 310, 261 (1986).

[23] F. Weber, Prog. Part. Nucl. Phys. 54, 193 (2005), astro-ph/0407155.

[24] E. A. Baltz, eConf C040802, L002 (2004), astro-ph/0412170.

[25] R. C. Tolman, Phys. Rev. 55, 364 (1939).

[26] R. C. Tolman, Relativity, Thermodynamics and Cosmology (Oxford University Press, Oxford, 1934).

[27] S. Weinberg, Gravitation and Cosmology: Principles and Applications of the General Theory of Relativity (John Wiley and Sons, New York, 1972).

[28] C. W. Misner, K. S. Thorne, and J. A. Wheeler, Gravitation (W. H. Freeman and Company, New York, 1973).

[29] B. K. Harrison, K. S. Thorne, M. Wakano, and J. A. Wheeler, Gravitation Theory and Gravitational Collapse (The Unversity of Chicago Press, Chicago, 1965).

[30] N. Straumann, General relativity with applications to astrophysics (Springer, Berlin, 2004).

[31] E. S. Fraga, R. D. Pisarski, and J. Schaffner-Bielich, Phys. Rev. D 63, 121702(R) (2001), hep-ph/0101143.

[32] G. Baym and S. A. Chin, Phys. Lett. B62, 241 (1976).

[33] I. N. Mishustin, L. M. Satarov, H. Stöcker, and W. Greiner, Phys. Rev. C 66, 015202 (2002), hep-ph/0203241.

[34] T. Padmanabhan, Theoretical Astrophysics, Volume II: Stars and Stellar Structure (Cambridge University Press, Cambridge, 2001).

[35] S. N. Ahmed et al. (SNO), Phys. Rev. Lett. 92, 181301 (2004), nucl-ex/0309004.

[36] D. N. Spergel et al. (WMAP), Astrophys. J. Suppl. 148, 175 (2003), astro-ph/0302209.

[37] A. D. Dolgov and S. H. Hansen, Astropart. Phys. 16, 339 (2002), hep-ph/0009083.

[38] A. Bottino, F. Donato, N. Fornengo, and S. Scopel, Phys. Rev. D 72, 083521 (2005), hep-ph/0508270.

[39] G. Börner, The early universe: Facts and fiction (Springer, Berlin, 2004).

[40] K. Rajagopal, M. S. Turner, and F. Wilczek, Nucl. Phys. B 358, 447 (1991). 
[41] L. Covi, J. E. Kim, and L. Roszkowski, Phys. Rev. Lett. 82, 4180 (1999), hep-ph/9905212.

[42] M. Y. Khlopov and C. A. Stephan (2006), astro-ph/0603187.

[43] M. A. Markov, Phys. Lett. 10, 122 (1964).

[44] J. G. Gao and R. Ruffini, Phys. Lett. 97B, 388 (1980).

[45] N. Bilic, G. B. Tupper, and R. D. Viollier, Lect. Notes Phys. 616, 24 (2003), hep-ph/0203039.

[46] N. Bilic, G. B. Tupper, and R. D. Viollier (2003), astro-ph/0310294.

[47] N. Tetradis, Phys. Lett. B632, 463 (2006), hep-ph/0507288.

[48] N. Brouzakis and N. Tetradis, JCAP 0601, 004 (2006), astro-ph/0509755.

[49] V. Koch, Int. J. Mod. Phys. E6, 203 (1997), nucl-th/9706075.

[50] B. D. Serot and J. D. Walecka, Adv. Nucl. Phys. 16, 1 (1986).

[51] M. Hanauske, L. M. Satarov, I. N. Mishustin, H. Stöcker, and W. Greiner, Phys. Rev. D 64, 043005 (2001).

[52] Y. B. Zel'dovich, Zh. Eksp. Teoret. Fiz. 41, 1609 (1961).

[53] Y. B. Zel'dovich and I. D. Novikov, Relativistic astrophysics. Volume 1: Stars and relativity (University of Chicago Press, Chicago, 1971).

[54] J. M. Lattimer and M. Prakash, Astrophys. J. 550, 426 (2001), astro-ph/0002232.

[55] M. Colpi, S. L. Shapiro, and I. Wasserman, Phys. Rev. Lett. 57, 2485 (1986).

[56] C. Alcock et al., Astrophys. J. 550, L169 (2001). 